\title{
Incidence, risk factors and prognostic impact of acute kidney injury after coronary angiography and intervention in kidney transplant recipients: a single-center retrospective analysis
}

\author{
Jeannine Lang, Sammy Patyna, Stefan Büttner, Helge Weiler, Helmut Geiger, Ingeborg Hauser, \\ Mariuca Vasa-Nicotera, Andreas M. Zeiher, Stephan Fichtlscherer, Jörg Honold \\ Department of Internal Medicine III, Division of Cardiology and Nephrology, University Hospital, Frankfurt, Germany
}

Adv Interv Cardiol 2020; 16, 1 (59): 58-64

DOI: https://doi.org/10.5114/aic.2020.93913

\begin{abstract}
A bstract
Introduction: Kidney transplant recipients (KTR) represent a high-risk population for cardiovascular disease. Coronary artery disease (CAD) is the most common cause of morbidity and mortality in this population. In KTR, coronary angiography and intervention (CI) can be associated with the risk of acute kidney injury (AKI).

Aim: Data about the incidence and impact of AKI after $\mathrm{Cl}$ in this population are rare. The aim of the present study is to describe the incidence and risk factors of AKI, periprocedural bleeding and the prognostic impact on 1-year mortality in KTR undergoing $\mathrm{Cl}$.

Material and methods: This retrospective single-center study includes all KTR undergoing $\mathrm{Cl}$ at University Hospital Frankfurt between 2005 and 2015.

Results: A total of $135 \mathrm{Cls}$ in KTR were analyzed. AKI occurred in 31 of $135 \mathrm{Cls}$ (23\%, AKI group). Patients of the AKI group were older; other baseline characteristics did not show significant differences. The amount of contrast dye used was higher in the AKI group ( $p=$ NS). Periprocedural bleeding defined by BARC criteria occurred more often in the AKI group ( $23 \%$ vs. $5 \%, p<0.01)$ and persisted as a risk factor of AKI in multivariate analysis (odds ratio $=6.43,95 \% \mathrm{Cl}: 1.78-23.20, p=0.01$ ). In-hospital mortality was $3 \%$ in the AKI group; no patient of the non-AKI group died during hospitalization $(p=0.2)$. One-year-survival was significantly higher in the non-AKI group ( $94 \%$ vs. $81 \%, p=0.02$ ).

Conclusions: AKI is an important prognostic determinant in KTR undergoing coronary angiography and percutaneous coronary intervention ( $\mathrm{PCI}$ ). Periprocedural bleeding events were associated with AKI. Well-known risk factors for AKI such as contrast agent and diabetes were of minor impact.
\end{abstract}

Key words: coronary artery disease, acute kidney injury, percutaneous coronary intervention, coronary angiography, coronary intervention, kidney transplant recipients.

Su m m a ry

Kidney transplant recipients (KTR) represent a high-risk population for cardiovascular disease and often have to undergo coronary intervention $(\mathrm{CI})$, which can be associated with the risk of acute kidney injury (AKI). This retrospective single-center study presents the incidence and outcome of AKI after Cl, analyzing $135 \mathrm{Cls}$ in KTR between 2005 and 2015 at University Hospital Frankfurt. The incidence of AKI was 23\%; 1-year-survival was significantly higher in the non-AKI group ( $94 \%$ vs. $81 \%, p=0.02$ ). AKI was associated with periprocedural bleeding events, whereas well-known risk factors such as contrast agent or diabetes were of minor impact in our study population.

\section{Introduction}

Kidney transplant recipients (KTR) undergoing invasive coronary angiography and percutaneous coronary intervention $(\mathrm{Cl})$ represent a high-risk cohort in different ways:
Both incidence and severity of coronary artery disease are more pronounced in patients suffering from chronic kidney disease (CKD) [1-3]. KTR often experienced long-term renal replacement therapy (RRT) while

\section{Corresponding author:}

PD Jörg Honold, Department of Internal Medicine III, Division of Cardiology and Nephrology, University Hospital, Frankfurt, Germany,

e-mail: jhonold@joho.de

Received: 18.06.2019, accepted: 3.10.2019. 
awaiting kidney transplantation with a known adverse impact on atherosclerotic burden and a high coincidence of cardiovascular risk factors such as diabetes or hypertension [1, 4, 5]. Even after successful transplantation, cardiovascular disease is highly prevalent in this population and the most frequent cause of death in KTR [3-6].

When performing $\mathrm{Cl}$ in $\mathrm{KTR}$, the interventional cardiologist is often faced with a more severe grade of coronary artery disease (CAD) with calcified lesions bearing a higher risk of prolonged $\mathrm{Cl}$ procedures and procedural complications, necessitating larger volumes of nephrotoxic contrast dye than standard procedures.

The accurate choice of arterial access is an utmost safety issue for every patient undergoing coronary angiography and $\mathrm{Cl}$ [7-10]. In recent years, the transradial access has gained a IA recommendation in the guidelines due to reduced bleeding rates translating into improved survival [11]. However, end-stage CKD and KTR are still often deemed unsuitable for transradial access as the risk of an irreversible injury of the radial artery as the potential donor vessel for dialysis shunt in the future is judged as too high $[11,12]$.

The transplanted kidney often displays reduced clearing function and higher vulnerability to contrast dye due to various reasons: sympathetic denervation during explantation impairs the kidney vessels' autoregulation, calcineurin inhibitors are known to have adverse effects on donor kidneys [13-15], and the donor organ might have been affected by pre- and peri-transplant organ handling as well as subclinical presence of chronic renal impairment before transplantation [16].

\section{Aim}

The aim of the present study is to describe the incidence and risk factors of AKI, periprocedural bleeding and their prognostic impact on 1-year mortality in KTR undergoing $\mathrm{Cl}$ in a retrospective, single-center analysis. The primary endpoint was acute kidney injury defined by the AKIN classification (as described in the methods section). Secondary endpoints were periprocedural bleeding events as defined by the BARC classification and 1-year survival.

\section{Material and methods}

\section{Patients' data collection and follow-up}

For this single-center retrospective analysis, all patients with a history of kidney transplantation who underwent coronary angiography with or without $\mathrm{PCl}$ were selected according to the ICD-10 code for kidney transplantation and OPS codes for invasive coronary diagnostics and $\mathrm{PCl}$ at Goethe University Hospital Frankfurt, Germany between 2005 and 2015. For every patient, demographic, clinical, laboratory and procedural data were collected from the electronic hospital information system (Orbis, Agfa). Glomerular filtration rate was estimated according the MDRD formula with the creatinine value at baseline. Follow-up data were collected from the electronic patient files, affiliated outpatient clinics and requests from the patients' general practitioners. Patients were followed up from the day of index procedure until 12 months after or all-cause death.

This study complies with the local standards of the institutional ethics committee (Approval file No. 312/16) and the Helsinki Declaration from 1975.

\section{Classification of kidney injury}

Serum creatinine value and glomerular filtration rate estimated by the 4-variable MDRD formula (eGFR) were used as baseline kidney function. Acute kidney injury was defined according to the AKIN classification (Acute Kidney Injury Network) [17]. AKIN 1 is defined as an increase of serum creatinine of $>0.3 \mathrm{mg} / \mathrm{dl}$ or a 1.5 to 2 -fold increase from baseline. AKIN 2 is defined as an increase of 2 to 3-fold and AKIN 3 as an increase of more than 3 -fold or creatinine $>4 \mathrm{mg} / \mathrm{dl}$ with an acute increase of $>0.5 \mathrm{mg} / \mathrm{dl}$.

\section{Classification of periprocedural bleeding events}

Complications due to bleeding were defined by the BARC criteria (Bleeding Academic Research Consortium) [18]. Type 0 means no signs of bleeding. Type 1 bleeding does not require any treatment. Overt signs of hemorrhage without the need for action are defined as type 2 . Type 3 bleeding is divided as follows: type $3 a-$ overt bleeding with hemoglobin drop $3.5 \mathrm{~g} / \mathrm{dl}+$ transfusion; type $3 \mathrm{~b}$ hemoglobin drop $>5 \mathrm{~g} / \mathrm{dl} /$ cardiac tamponade/ need for surgical intervention/IV vasoactive agents; type $3 c$ intracranial bleeding. Any coronary artery bypass grafting (CABG)-related bleeding is classified as type 4, and fatal bleeding is classified as type 5 .

\section{Statistical analysis}

Statistical analyses were performed using Prism 7 (GraphPad Software Inc.; San Diego, USA) and SPSS 23.0 (IBM). Continuous variables are shown as median and interquartile range (IQR), while categorical variables are reported as frequencies and percentages. Differences between patient cohorts were determined using Fisher's exact test for categorical variables; for quantitative variables the Mann-Whitney- $U$ test was used. The Kaplan-Meier estimator and log-rank test were used for survival analysis. Predictors of survival and endpoints were determined with Cox regression analysis. Binary univariate and multivariate logistic regression analyses were performed to study the determinants of independent predictors of AKI. Odds ratio (OR) and corresponding $95 \%$ confidence intervals were calculated simultaneously. A two-sided $p$-value of $<0.05$ was considered significant. 


\section{Results}

\section{Patients}

As depicted in Table I, patients were predominantly male ( $79 \%$ male gender), with a median age of 65 years, a high burden of preexisting chronic transplant kidney disease (median serum creatinine $1.8 \mathrm{mg} / \mathrm{dl}$ ) and a high prevalence of diabetes mellitus (36\%) and coronary multivessel disease $(72 \%)$. Left ventricular ejection fraction - LVEF) was preserved in the majority of patients.
During the study period, 135 invasive coronary procedures were performed in our institution (see Table I for details). Of those, 53 (39\%) procedures were diagnostic. In $82(61 \%)$ procedures, $\mathrm{PCl}$ of one or more coronary arteries was performed. The indication for the invasive approach was predominantly elective (69\%) while $31 \%$ were urgent indications in the setting of acute coronary syndromes. Transfemoral access was performed in 93\% of the procedures, while $7 \%$ of the procedures were performed via transradial access.

Table I. Baseline characteristics, indications and complications after coronary angiography of AKI vs. non-AKI group

\begin{tabular}{|c|c|c|c|c|}
\hline Parameter & All $(n=135)$ & AKI $(n=31)$ & Non-AKI $(n=104)$ & $P$-value \\
\hline \multicolumn{5}{|l|}{ Baseline characteristics: } \\
\hline Age, (IQR) [years] & $65(56-71)$ & $68(65-74)$ & $64(55-71)$ & 0.01 \\
\hline Gender male, $n(\%)$ & $106(79)$ & $22(71)$ & $84(81)$ & 0.32 \\
\hline Baseline creatinine, (IQR) [mg/dl] & $1.8(1.5-6.9)$ & $2.0(1.6-5.1)$ & $1.8(1.4-6.9)$ & 0.12 \\
\hline GFR, (IQR) [ml/min] & $34(26-48)$ & $32(24-53)$ & $36(27-52)$ & $<0.05$ \\
\hline Median EF, \% (IQR) & $60(54-65)$ & $60(43-65)$ & $60(55-65)$ & 0.13 \\
\hline Diabetes mellitus, $n(\%)$ & $49(36)$ & $15(48)$ & $34(33)$ & 0.14 \\
\hline \multicolumn{5}{|l|}{ Renal disease, $n(\%)$ : } \\
\hline Chronic glomerulonephritis & $65(48)$ & $19(62)$ & $46(44)$ & 0.11 \\
\hline Diabetic kidney disease & $8(6)$ & $2(6)$ & $6(6)$ & 0.99 \\
\hline Polycystic kidney disease & $21(16)$ & $4(13)$ & $17(16)$ & 0.78 \\
\hline Other & $41(30)$ & $6(19)$ & $35(34)$ & 0.18 \\
\hline \multicolumn{5}{|l|}{ Immunosuppression, $n(\%)$ : } \\
\hline Cyclosporin & $33(24)$ & $10(32)$ & $23(22)$ & 0.34 \\
\hline Tacrolimus & $61(45)$ & $10(32)$ & $51(49)$ & 0.11 \\
\hline Mycophenolate mofetil/acid & $50(37)$ & $15(48)$ & $35(34)$ & 0.14 \\
\hline \multicolumn{5}{|l|}{ Indications and characteristics: } \\
\hline Acute coronary syndrome, $n(\%)$ & $42(31)$ & & $28(27)$ & 0.08 \\
\hline Elective CA, $n(\%)$ & $93(69)$ & & $76(73)$ & 0.08 \\
\hline Diagnostic CA, $n$ (\%) & $53(39)$ & & $40(38)$ & 0.83 \\
\hline $\mathrm{PCl}, n(\%)$ & $82(61)$ & & $64(62)$ & 0.83 \\
\hline Previous PCI, $n(\%)$ & $75(56)$ & & $61(59)$ & 0.22 \\
\hline Transfemoral access, $n(\%)$ & $126(93)$ & & $96(92)$ & 0.68 \\
\hline Transradial access, $n(\%)$ & $9(7)$ & & $8(8)$ & 0.68 \\
\hline Contrast agent, (IQR) [ml] & $130(70-200)$ & & $127(71-200)$ & 0.43 \\
\hline Fluoroscopy time, (IQR) [min] & $11(5-22)$ & & $10(5-19)$ & 0.16 \\
\hline \multicolumn{5}{|l|}{ Findings CA, $n(\%)$ : } \\
\hline No CHD & $16(12)$ & & $12(12)$ & 0.76 \\
\hline 1-vessel CHD & $22(16)$ & & $15(14)$ & 0.28 \\
\hline 2-vessel CHD & $31(23)$ & & $27(26)$ & 0.15 \\
\hline 3-vessel CHD & $66(49)$ & & $50(48)$ & 0.84 \\
\hline \multicolumn{5}{|l|}{ Perioperative complications, $n(\%)$ : } \\
\hline Bleeding [BARC] & $12(9)$ & & $5(5)$ & $<0.01$ \\
\hline Myocardial infarction & $0(0)$ & & $0(0)$ & $>0.99$ \\
\hline Stroke & $2(1)$ & & $1(1)$ & 0.41 \\
\hline Need for surgery & $3(2)$ & & $1(1)$ & 0.13 \\
\hline CPR & $1(1)$ & & $0(0)$ & 0.23 \\
\hline Death & $1(1)$ & & $0(0)$ & 0.23 \\
\hline
\end{tabular}




\section{Acute kidney injury}

AKI occurred after 31 of the 135 procedures, representing an incidence of $23 \%$. These two groups are named "AKI" and "non-AKI" below. AKIN 1 was observed in $77 \%$ of the AKI patients, AKIN 3 with the need for transient RRT in $23 \%$ of the AKI group (Table II). No patient developed AKIN 3 with permanent RRT during the study period. There was no case of AKIN 2. No significant differences in the incidence of AKI were found after diagnostic angiography $(25 \%)$ or $\mathrm{PCl}(22 \% ; p=0.83)$. Furthermore, the incidence of AKI after transfemoral access (24\%) and the incidence of AKI after transradial access (11\%) did not show a significant difference $(p=0.68)$. Patients from the AKI group were significantly older (Table I) and displayed a higher proportion of acute coronary syndromes as the indication for invasive approach ( $p=$ NS, Table I). Further, AKI patients suffered more often from diabetes mellitus and coronary multivessel disease, without reaching statistical significance for these parameters. The total amount of contrast dye was not significantly higher in the AKI group (155 ml (IQR: 70-225) vs. $127 \mathrm{ml}$ (IQR: 71-200), $p=0.43$ ).

There was no difference in percentages of male patients in the AKI and non-AKI group (71\% vs. $81 \%$, $p=0.32$ ). Patients of the AKI group tended to have higher creatinine levels at baseline $(2.0 \mathrm{mg} / \mathrm{dl}$ vs. $1.8 \mathrm{mg} / \mathrm{dl}$, $p=0.12)$ and significantly lower eGFR $(32 \mathrm{ml} / \mathrm{min}$ vs. $36 \mathrm{ml} / \mathrm{min} ; p \leq 0.05$ ). Most of the patients suffered from chronic glomerulonephritis as chronic kidney disease (62\% in the AKI group and $44 \%$ in the non-AKI group; $p=0.11$ ). There were no significant differences in the immunosuppression scheme between the groups.

In multivariate analysis, periprocedural bleeding persisted as a single independent predictor of AKI with an OR of 6.43 (95\% Cl: 1.78-23.20, $p=0.01$; Table III).

\section{Periprocedural bleeding complications}

Bleeding events were registered throughout hospitalization; bleeding events after hospital discharge have not been recorded. There were 10 patients with access
Table II. Incidence and extent of acute kidney injury, need for acute hemodialysis and graft loss

\begin{tabular}{lcc} 
Parameter & All $(n=135)$ & AKI $(n=31)$ \\
\hline AKIN 1-3, $n(\%):$ & $31(23)$ & $31(100)$ \\
\hline AKIN 1 & $24(18)$ & $24(77)$ \\
\hline AKIN 2 & $0(0)$ & $0(0)$ \\
\hline AKIN 3 & $7(5)$ & $7(23)$ \\
\hline Need for acute hemodialysis & $7(5)$ & $7(23)$ \\
\hline Graft loss & $0(0)$ & $0(0)$
\end{tabular}

site bleeding, 2 patients with pericardial tamponade. Periprocedural bleeding as defined by the BARC criteria occurred in $23 \%$ of the AKI group and only $5 \%$ of the non-AKI group $(p<0.01)$. Bleedings were mainly categorized as type $3 a$ or $3 b$ (see Classification of periprocedural bleeding events and Figure 1). Access-site bleeding occurred only in patients with femoral access. An extended univariate analysis was performed for the secondary endpoint without detecting predictors of periprocedural bleeding. Surgical treatment after access site bleeding was necessary for 2 patients of the AKI group and 1 patient of the non-AKI group.

\section{Mortality and follow-up}

In-hospital mortality was $3 \%$ in the AKI group; no patient of the non-AKI group died during hospitalization $(p=0.2)$. One-year survival was $81 \%$ in the AKI group and $94 \%$ in the non-AKI group ( $p=0.02$; Figure 2 ).

Kidney function was analyzed for 1, 6 and 12 months after $\mathrm{Cl}$. Kidney function was stable during follow-up. Figure 3 shows serum creatinine values and IQR 30 days, 6 and 12 months after $\mathrm{Cl}$. There was no significant difference in serum creatinine after 12 months $(p=0.28)$.

In multivariate analysis, only the occurrence of periprocedural bleeding complications persisted as a risk factor of acute kidney injury in these patients (Table III).

Table III. Predictors and potential risk factors for development of AKI - univariate and multivariate analysis for endpoint AKI

\begin{tabular}{|c|c|c|c|c|c|c|}
\hline \multirow[t]{2}{*}{ Parameter } & \multicolumn{3}{|c|}{ Univariate analysis } & \multicolumn{3}{|c|}{ Multivariate analysis } \\
\hline & Odds ratio & $95 \% \mathrm{Cl}$ & $P$-value & Odds ratio & $95 \% \mathrm{Cl}$ & $P$-value \\
\hline Age & 1.04 & $1-1.10$ & $<0.05$ & & & \\
\hline Contrast agent & 1.00 & 0.99-1.01 & 0.44 & & & \\
\hline Fluoroscopy time & 1.03 & $0.99-1.06$ & 0.11 & & & \\
\hline Diabetes mellitus & 1.65 & $0.71-3.80$ & 0.24 & 2.06 & $0.86-4.91$ & 0.10 \\
\hline Creatinine & 1.17 & $0.80-1.73$ & 0.42 & 1.24 & $0.82-1.88$ & 0.31 \\
\hline Periprocedural bleeding & 4.65 & $1.29-16.71$ & 0.02 & 6.43 & $1.78-23.20$ & 0.01 \\
\hline
\end{tabular}




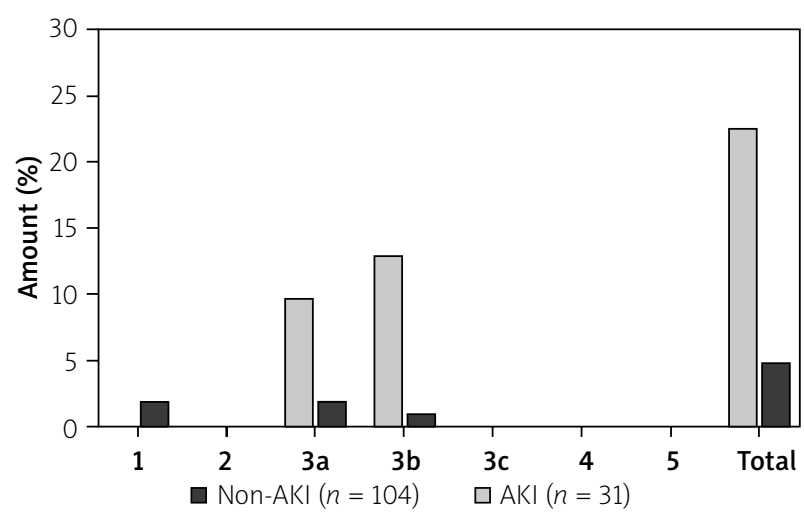

Figure 1. Incidence of bleeding complications defined by BARC criteria in \%

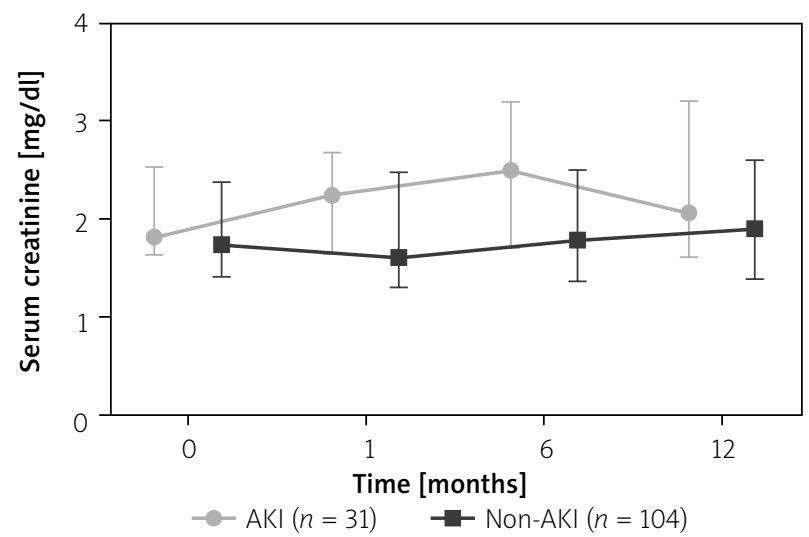

Figure 3. Follow-up of serum creatinine levels - AKI vs. non-AKI after 1, 6 and 12 months

The amount of contrast dye used during the $\mathrm{Cl}$ was not an independent predictor of acute kidney injury in this study.

\section{Discussion}

The accurate choice of coronary revascularization technique in KTR remains a complex task [19-22]. In patients undergoing invasive coronary angiography and $\mathrm{PCl}$, acute kidney injury following coronary angiography and $\mathrm{PCl}$ is a topic of high interest. This study of kidney transplant recipients receiving $\mathrm{Cl}$ analyzed the incidence and outcome of acute kidney injury and interaction with periprocedural bleeding.

In our study, the incidence of AKI after CI was 23.0\%; no patient needed permanent RRT. Our results are comparable to several studies regarding the incidence of AKI in KTR: Lees et al. found an incidence of 18.9\% [23], Neves et al. reported an incidence of AKI of $17.5 \%$ in 7800 patients after coronary angiography [24]. Fähling et al. found an incidence of $30 \%$ for contrast-induced nephropathy [25]. Victor et al. indicated the amount of contrast dye as an important risk factor for AKI and included it in their risk scoring model together with GFR, diabetes, anemia, hypotension and peripheral artery disease [26].

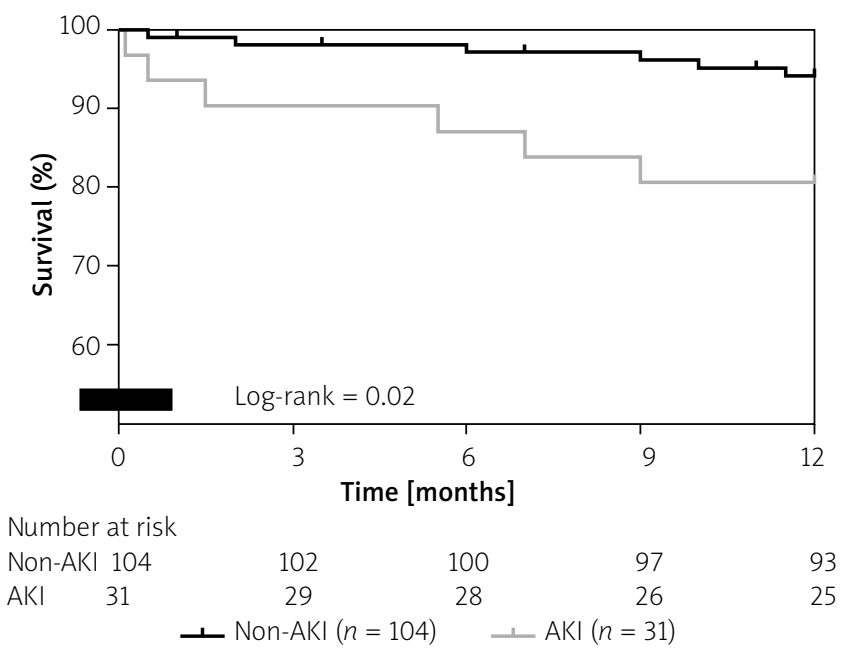

Figure 2. Survival at 3, 6 and 12 months after coronary angiography

In order to avoid AKI in patients with renal disease undergoing $\mathrm{Cl}$, advanced $\mathrm{PCl}$ technologies to reduce contrast dye are evaluated such as ultra-low contrast angiography and zero-contrast PCI [27]. Ali et al. performed Cl without contrast in 31 patients with advanced CKD using IVUS, without changes in creatinine levels or eGFR in the follow-up period [28]. The study of Sacha et al. showed that zero-contrast PCI was a safe and effective method in 20 patients with severe CKD undergoing 29 procedures and also reduced the estimated risk for AKI from $26 \%$ to a prevalence of $10 \%$ without specific complications [29].

However, Caspi et al. found similar rates of AKI in patients with STEMI with and without contrast exposure [30], indicating the presence of several factors for development of AKI in cardiovascular patients: age over 70 years, GFR, diabetes mellitus and heart failure [30].

In our study, univariate analysis indicated that the amount of contrast dye was not a significant risk factor for development of AKI; both the AKI and non-AKI group received substantial amounts of dye during $\mathrm{Cl}$. However, we found periprocedural bleeding complications to be associated significantly with acute kidney injury. Our findings correspond to those of Neves et al., who found major bleeding as a risk factor for AKI with higher in-hospital mortality and lower 1-year survival [24]. The vast majority of $\mathrm{PCl}$ in our study were performed using a transfemoral access. Several studies have described the association of arterial access and AKI in terms of less AKI in patients with transradial access (TRA). TRA is associated with significantly reduced rates of major bleeding [31]. Whether the pathophysiological mechanism for increased AKI rates in patients with major bleeding is caused by hemodynamic changes in terms of hypovolemic shock under bleeding or a loss of hemoglobin remains unclear [32]. In the study of Pancholy et al., TRA was associated with a $43 \%$ relative reduction of post- $\mathrm{PCI}$ AKI compared to transfemoral access [33]. 
Pathogenesis of $\mathrm{AKI}$ in patients suffering from bleeding after $\mathrm{Cl}$ may involve several pathways: first, acute hypovolemic shock due to bleeding will induce shock-induced nephropathy. Second, the need for transfusion after bleeding also has a negative impact on kidney function. The paper by Ohno et al. describes an increased risk of AKI also in patients with a drop in hemoglobin levels without hemodynamic change [34]. Third, the possibility of catheter-induced microembolization into the transplant donor vessel during aortal catheter passage has to be taken into account in patients with transfemoral access [35].

This study bears several important limitations: first, the lack of information about intraprocedural hemodynamics in patients with and without AKI. Moreover, serum creatinine could not be analyzed for all patients during the follow-up; only one patient underwent $\mathrm{Cl}$ in cardiogenic shock. Further, this study is limited by its design as a retrospective observational study. Hence, we could not describe independent risk factors of periprocedural bleeding due to the non-randomized study fashion with predominant transfemoral arterial access and a small absolute number of bleeding events. Therefore, we only can speculate that improved access safety in terms of more frequent use of transradial access in a comparable KTR population will be associated with fewer bleeding events, less AKI and improved survival.

\section{Conclusions}

Cardiovascular disease is the most common cause of morbidity and mortality in patients with impaired kidney function. This study analyzed the incidence, risk factors and outcome of acute kidney injury and bleeding complications in kidney transplant recipients receiving coronary interventions in diagnostic or therapeutic intention. Kidney transplant recipients of our cohort with acute kidney injury after coronary intervention showed lower 1-year-survival. Graft function was stable 12 months after the procedure. In this subset of patients at high risk for AKI receiving substantial amounts of contrast dye for $\mathrm{Cl}$, periprocedural bleeding could be identified as an important independent risk factor for AKI. Optimized vascular access and the avoidance of periprocedural bleeding complications seem to be essential in this high-risk population.

\section{Acknowledgments}

We would like to thank Mrs Maier and Mrs Mentges, as well as the cardiac catheter laboratory staff and the study nurses, for their support and assistance.

\section{Conflict of interest}

The authors declare no conflict of interest.

\section{References}

1. Levin A. The clinical epidemiology of cardiovascular diseases in chronic kidney disease: clinical epidemiology of cardiovascular disease in chronic kidney disease prior to dialysis. Semin Dial 2003; 16: 101-5.

2. Foley RN, Parfrey PS, Sarnak MJ. Clinical epidemiology of cardiovascular disease in chronic renal disease. Am J Kidney Dis 1998; 32: 112-9.

3. Paizis IA, Mantzouratou PD, Tzanis GS, et al. Coronary artery disease in renal transplant recipients: an angiographic study. Hellenic J Cardiol 2018 [Epub ahead of print]; doi: 10.1016/j. hjc.2018.07.002.

4. Banas MC, Banas B, Orth SR, et al. Traditional and nontraditional cardiovascular risk factors and estimated risk for coronary artery disease in renal transplant recipients: a single-center experience. Nephron Clin Pract 2011; 119: c227-35.

5. Seyahi N, Kahveci A, Cebi D, et al. Coronary artery calcification and coronary ischaemia in renal transplant recipients. Nephrol Dial Transplant 2011; 26: 720-6.

6. Pirat B, Müderrisoglu H, Korkmaz M, et al. Characteristics of coronary heart disease in renal transplant recipients. Transplant Proc 2004; 36: 152-5.

7. Bagai J, Little B, Banerjee S. Association between arterial access site and anticoagulation strategy on major bleeding and mortality: a historical cohort analysis in the Veteran population. Cardiovasc Revasc Med 2018; 19: 95-101.

8. Déry JP, Mahaffey KW, Tricoci P, et al. Arterial access site and outcomes in patients undergoing percutaneous coronary intervention with and without vorapaxar. Catheter Cardiovasc Interv 2016; 88: 163-73.

9. Saito S, Isshiki T, Kimura T, et al. Impact of arterial access route on bleeding complications in Japanese patients undergoing percutaneous coronary intervention - insight from the PRASFIT Trial. Circ J 2015; 79: 1928-37.

10. Mamas MA, Anderson SG, Carr M, et al. Baseline bleeding risk and arterial access site practice in relation to procedural outcomes after percutaneous coronary intervention. J Am Coll Cardiol 2014; 64: 1554-64.

11. Hamon M, Pristipino C, Di Mario C, et al. Consensus document on the radial approach in percutaneous cardiovascular interventions: position paper by the European Association of Percutaneous Cardiovascular Interventions and Working Groups on Acute Cardiac Care ${ }^{* *}$ and Thrombosis of the European Society of Cardiology. Eurolntervention 2013; 8: 1242-51.

12. Koutouzis M, Sfyroeras GS, Maniotis C, et al. Forearm versus femoral approach for cardiac catheterization in end-stage renal disease patients. J Invasive Cardiol 2018; 30: 110-4.

13. Rao SV, Tremmel JA, Gilchrist IC, et al. Best practices for transradial angiography and intervention: a consensus statement from the society for cardiovascular angiography and intervention's transradial working group. Catheter. Cardiovasc Interv 2014; 83: 228-36.

14. Gooch JL, King C, Francis CE, et al. Cyclosporine A alters expression of renal microRNAs: new insights into calcineurin inhibitor nephrotoxicity. PLoS One 2017; 12: e0175242.

15. Akhtar T, Sheikh N, Shan T, et al. Tacrolimus induced nephrotoxicity and pulmonary toxicity in Wistar rats. J Biol Regul Homeost Agents 2017; 31: 1061-6.

16. Guimarães J, Araújo AM, Santos F, et al. Living-donor and deceased-donor renal transplantation: differences in early outcome - a single-center experience. Transplant Proc 2015; 47: 958-62.

17. Mehta RL, Kellum JA, Shah SV, et al. Acute Kidney Injury Network: report of an initiative to improve outcomes in acute kidney injury. Crit Care 2007; 11: R31. 
18. Mehran R, Rao SV, Bhatt DL, et al. Standardized bleeding definitions for cardiovascular clinical trials. Circulation 2011; 123 : 2736-47.

19. Lang J, Buettner S, Weiler $\mathrm{H}$, et al. Comparison of interventional and surgical myocardial revascularization in kidney transplant recipients - a single-centre retrospective analysis. IJC Hear VasC 2018; 21: 96-102.

20. Lenihan CR, Montez-Rath ME, Winkelmayer WC, et al. Drug-eluting stents versus bare metal stents for percutaneous coronary intervention in kidney transplant recipients. Transplantation 2017; 101: 851-7.

21. Lemos PA, Arampatzis CA, Hoye A, et al. Impact of baseline renal function on mortality after percutaneous coronary intervention with sirolimus-eluting stents or bare metal stents. Am J Cardiol 2005; 95: 167-72.

22. Herzog CA, Ma JZ, Collins AJ. Long-term outcome of renal transplant recipients in the united states after coronary revascularization procedures. Circulation 2004; 109: 2866-71.

23. Lees JS, Findlay MD, Mark PB, et al. The impact of coronary angiography on renal transplant function. QJM An Int J Med 2019; 112: 23-7.

24. Neves D, Belo A, Damásio AF, et al. Acute kidney injury in acute coronary syndromes - an important multifactorial consequence. Rev Port Cardiol 2016; 35: 415-21.

25. Fähling $M$, Seeliger $E$, Patzak A, et al. Understanding and preventing contrast-induced acute kidney injury. Nat Rev Nephrol 2017; 13: 169-80.

26. Victor SM, Gnanaraj A, S. V, et al. Risk scoring system to predict contrast induced nephropathy following percutaneous coronary intervention. Indian Heart J 2014; 66: 517-24.

27. Sacha J, Gierlotka M, Feusette P, et al. Ultra-low contrast coronary angiography and zero-contrast percutaneous coronary intervention for prevention of contrast-induced nephropathy: step-bystep approach and review. Adv Interv Cardiol 2019; 15: 127-36.

28. Ali ZA, Galougahi KK, Nazif T, et al. Imaging- and physiologyguided percutaneous coronary intervention without contrast administration in advanced renal failure: a feasibility, safety, and outcome study. Eur Heart J 2016; 37: 3090-5.

29. Sacha J, Gierlotka M, Lipski P, et al. Zero-contrast percutaneous coronary interventions to preserve kidney function in patients with severe renal impairment and hemodialysis subjects. Adv Interv Cardiol 2019; 15: 137-42.

30. Caspi O, Habib M, Cohen Y, et al. Acute kidney injury after primary angioplasty: is contrast-induced nephropathy the culprit? J Am Heart Assoc 2017; 6: pii: e005715.

31. Valgimigli M, Gagnor A, Calabró P, et al. Radial versus femoral access in patients with acute coronary syndromes undergoing invasive management: a randomised multicentre trial. Lancet 2015; 385: 2465-76.

32. Steinvil A, Garcia-Garcia HM, Rogers T, et al. Comparison of propensity score-matched analysis of acute kidney injury after percutaneous coronary intervention with transradial versus transfemoral approaches. Am J Cardiol 2017; 119: 1507-11.

33. Pancholy MS, Skelding K, Scott T, et al. Effect of access site choice on acute kidney injury after percutaneous coronary intervention. Am J Cardiol 2017; 120: 2141-5.

34. Ohno Y, Maekawa $\mathrm{Y}$, Miyata $\mathrm{H}$, et al. Impact of periprocedural bleeding on incidence of contrast-induced acute kidney injury in patients treated with percutaneous coronary intervention. J Am Coll Cardiol 2013; 62: 1260-6.

35. Scolari F, Ravani P. Atheroembolic renal disease. Lancet 2010; 375: 1650-60. 